\title{
Clonal hematopoiesis: background player in plasma cell-free DNA variants
}

\author{
Kun Sun \\ Shenzhen Bay Laboratory, Shenzhen 518055, China \\ Correspondence to: Kun Sun. Shenzhen Bay Laboratory, Shenzhen 518055, China. Email: sunkun@szbl.ac.cn. \\ Provenance: This is an invited article commissioned by the Section Editor Dr. Gang Xu (Department of Liver Surgery, Peking Union Medical College \\ Hospital, Chinese Academy of Medical Sciences and Peking Union Medical College, Beijing, China). \\ Comment on: Razavi P, Li BT, Brown DN, et al. High-intensity sequencing reveals the sources of plasma circulating cell-free DNA variants. Nat Med \\ 2019;25:1928-37.
}

Submitted Dec 05, 2019. Accepted for publication Dec 12, 2019.

doi: $10.21037 / \mathrm{atm} .2019 .12 .97$

View this article at: http://dx.doi.org/10.21037/atm.2019.12.97

The presence of cell-free tumor-derived DNA (ctDNA) in plasma of cancer patients was first reported 30 years ago (1), which opens the door of blood-based analysis for cancer diagnosis and monitoring, or so-called cancer liquid biopsy. In recent years, with the development of highthroughput and high sensitive techniques, cancer liquid biopsy has gained much interest and is actively researched globally (2). As a genetic disease, one hallmark of cancer is acquired somatic mutations in the genome of tumor cells, which could be detected in plasma ctDNA. A variety of assays had been developed to screen and quantify the tumor-derived somatic mutations in plasma. For instance, assays that utilized high depth sequencing technology, either on the whole human genome or specific regions of interest using target capture approaches, had showed good sensitivity and accuracy in this task $(3,4)$. However, parallel analysis of plasma DNA and matched tumor tissues had revealed that in cancer patients of various types, even though the somatic mutation profiles of these two materials showed remarkable concordance, they were not identical (4-6). Conventionally, the intratumor heterogeneity is proposed to be the major reason to this phenomenon $(2,7)$. However, the tissue origin of the mysterious somatic mutations that are only found in the plasma still remains elusive.

In a recent work published in Nature Medicine, Razavi and colleagues utilized a high-intensity sequencing assay of matched plasma DNA, tumor tissue and white blood cells for characterization and annotation of the somatic mutation repertoire in plasma DNA (8). A total of 124 cancer patients and 47 control subjects were involved in the study. With extra-high sequencing depth $(>60,000 \times)$ data generated using an assay that combined target capture and error-suppressive library preparation protocol, Razavi et al. identified 364 and 2,983 high-confidence variants in the plasma of control subjects and cancer patients, respectively. Interestingly, for the somatic mutations identified in plasma DNA of the cancer patients, only $24.4 \%$ of them also existed in the matched tumors. In addition, Razavi et al. found that an even higher proportion of the somatic mutations actually could be detected in the matched white blood cells of the cancer patients. With further validation analyses on the somatic mutations using various approaches, Razavi et al. concluded that the vast majority of plasma DNA mutations $(81.6 \%$ in controls and $53.2 \%$ in cancer patients), as a matter of fact, showed features that were consistent with clonal hematopoiesis. In fact, existence of somatic mutations in plasma DNA of non-cancer subjects had been reported before (2) and was linked to white blood cells in an earlier study by Liu et al. (9). Razavi et al. confirmed the findings in Liu et al. and extended the analysis to cancer patients, concluding that clonal hematopoiesis is a major contributor to plasma DNA variants, even in cancer patients. Razavi et al. work thus provided a convincing explanation to the discordance of somatic mutation profiles in plasma DNA and matched tumors in cancer patients.

Moreover, the tissue origin of the somatic mutations in plasma DNA is of high translational value in guiding the interpretation of cancer liquid biopsy results and 
optimization of assays to reduce false positives, since somatic mutation profile is one of the most widely used analyte in cancer liquid biopsy (10). Razavi et al. work thus proposed a conceptual guideline that the matched white blood cells should also be analyzed along with plasma DNA if the assay is based on screening somatic mutations. Notably, there is still a certain proportion of somatic mutations identified in plasma DNA only that could not be confidently characterized (8), i.e., they are not found in the tumors, white blood cells or any other tissues specifically linked to the patients' clinical scenario, which also had been reported in other studies using whole genome sequencing approach $(4,11)$. In most clinical scenarios, plasma cell-free DNA molecules mostly originates from the hematopoietic system $(12,13)$; however, this does not mean that they only come from the white blood cells. In fact, previous studies had revealed that in heathy subjects, DNA from the erythroid lineage could contribute as much as $30 \%$ of plasma cellfree DNA $(14,15)$. Clonal hematopoiesis also affects the erythroid lineage therefore may induce unique acquired somatic mutations that are different from white blood cells. It is therefore of interest to explore whether cells in the erythroid lineage is another contributor of plasma cellfree DNA variants besides white blood cells. Epigenetic features could also apply to trace the tissue source of the somatic mutations in plasma (16). For instance, one could profile the DNA methylation levels of the CpG sites in the plasma DNA containing a somatic mutation of interest then compare it to the methylomes of the tumor, the blood cells or other possible tissues for origination prediction. Considering that tumor-derived DNA is generally shorter than the background DNA in plasma (17), the size and cleavage pattern of the plasma DNA containing the somatic mutations could also provide clues to its tissue origin (18-20). Such analyses may further uncover new important players in plasma DNA variants.

In the meantime, Razavi et al. work has further demonstrated the clinical relevance of biology of plasma DNA to cancer liquid biopsy. Indeed, there is still much directions to be explored in plasma DNA, which knowledge could largely benefit our cancer liquid biopsy assays, including new concepts in diagnosis methods and guidelines for performance improvements. For instance, analysis of the tissue origin of plasma DNA could help us to predict the location of the tumor $(13,18,20)$, and tissue origin of the somatic mutations explains the false positives in various assays $(8,9)$. Another recent study had reported that Dnase1l3 gene is a newly-discovered essential player in plasma DNA fragmentation and its loss-of-function directly affects the motif profile of the plasma DNA in a mouse model (21); such discovery may lead to new approaches in fragmentomic analysis of plasma DNA $(19,22)$. In summary, Razavi et al. work revealed that besides the tumor, clonal hematopoiesis is another momentous player in plasma DNA variants, which enhanced our understanding and provides a guideline that could improve the performance of somatic mutation based cancer liquid biopsy assays.

\section{Acknowledgments}

None.

\section{Footnote}

Conflicts of Interest: The author has no conflicts of interest to declare.

Ethical Statement: The author is accountable for all aspects of the work in ensuring that questions related to the accuracy or integrity of any part of the work are appropriately investigated and resolved.

\section{References}

1. Stroun M, Anker P, Maurice P, et al. Neoplastic characteristics of the DNA found in the plasma of cancer patients. Oncology 1989;46:318-22.

2. Wan JCM, Massie C, Garcia-Corbacho J, et al. Liquid biopsies come of age: towards implementation of circulating tumour DNA. Nat Rev Cancer 2017;17:223-38.

3. Phallen J, Sausen M, Adleff V, et al. Direct detection of early-stage cancers using circulating tumor DNA. Sci Transl Med 2017;9:eaan2415.

4. Jiang P, Sun K, Tong YK, et al. Preferred end coordinates and somatic variants as signatures of circulating tumor DNA associated with hepatocellular carcinoma. Proc Natl Acad Sci U S A 2018;115:E10925-33.

5. Janku F, Zhang S, Waters J, et al. Development and Validation of an Ultradeep Next-Generation Sequencing Assay for Testing of Plasma Cell-Free DNA from Patients with Advanced Cancer. Clin Cancer Res 2017;23:5648-56.

6. Zhang X, Zhao W, Wei W, et al. Parallel Analyses of Somatic Mutations in Plasma Circulating Tumor DNA (ctDNA) and Matched Tumor Tissues in Early-Stage Breast Cancer. Clin Cancer Res 2019;25:6546-53. 
7. Gerlinger M, Rowan AJ, Horswell S, et al. Intratumor heterogeneity and branched evolution revealed by multiregion sequencing. N Engl J Med 2012;366:883-92.

8. Razavi P, Li BT, Brown DN, et al. High-intensity sequencing reveals the sources of plasma circulating cellfree DNA variants. Nat Med 2019;25:1928-37.

9. Liu J, Chen X, Wang J, et al. Biological background of the genomic variations of cf-DNA in healthy individuals. Ann Oncol 2019;30:464-70.

10. Cohen JD, Li L, Wang Y, et al. Detection and localization of surgically resectable cancers with a multi-analyte blood test. Science 2018;359:926-30.

11. Chan KCA, Jiang P, Sun K, et al. Second generation noninvasive fetal genome analysis reveals de novo mutations, single-base parental inheritance, and preferred DNA ends. Proc Natl Acad Sci U S A 2016;113:E8159-68.

12. Lui YYN, Chik KW, Chiu RWK, et al. Predominant hematopoietic origin of cell-free DNA in plasma and serum after sex-mismatched bone marrow transplantation. Clin Chem 2002;48:421-7.

13. Sun K, Jiang P, Chan KCA, et al. Plasma DNA tissue mapping by genome-wide methylation sequencing for noninvasive prenatal, cancer, and transplantation assessments. Proc Natl Acad Sci U S A 2015;112:E5503-12.

14. Moss J, Magenheim J, Neiman D, et al. Comprehensive human cell-type methylation atlas reveals origins of circulating cell-free DNA in health and disease. Nat

Cite this article as: Sun K. Clonal hematopoiesis: background player in plasma cell-free DNA variants. Ann Transl Med 2019;7(Suppl 8):S384. doi: 10.21037/atm.2019.12.97
Commun 2018;9:5068.

15. Lam WKJ, Gai W, Sun K, et al. DNA of erythroid origin is present in human plasma and informs the types of anemia. Clin Chem 2017;63:1614-23.

16. Gai W, Sun K. Epigenetic Biomarkers in Cell-Free DNA and Applications in Liquid Biopsy. Genes (Basel) 2019;10:32.

17. Underhill HR, Kitzman JO, Hellwig S, et al. Fragment length of circulating tumor DNA. PLoS Genet 2016;12:e1006162.

18. Sun K, Jiang P, Cheng SH, et al. Orientation-aware plasma cell-free DNA fragmentation analysis in open chromatin regions informs tissue of origin. Genome Res 2019;29:418-27.

19. Sun K, Jiang P, Wong AIC, et al. Size-tagged preferred ends in maternal plasma DNA shed light on the production mechanism and show utility in noninvasive prenatal testing. Proc Natl Acad Sci U S A 2018;115:E5106-14.

20. Snyder MW, Kircher M, Hill AJ, et al. Cell-free DNA comprises an in vivo nucleosome footprint that informs its tissues-of-origin. Cell 2016;164:57-68.

21. Serpas L, Chan RWY, Jiang P, et al. Dnase113 deletion causes aberrations in length and end-motif frequencies in plasma DNA. Proc Natl Acad Sci U S A 2019;116:641-9.

22. Cristiano S, Leal A, Phallen J, et al. Genome-wide cellfree DNA fragmentation in patients with cancer. Nature 2019;570:385-9. 